\title{
A Proposal for Inter-Domain QoS Routing based on Distributed Overlay Entities and QBGP ${ }^{1}$
}

\author{
Marcelo Yannuzzi ${ }^{1}$, Alexandre Fonte ${ }^{2,3}$, Xavier Masip-Bruin ${ }^{1}$, Edmundo Monteiro ${ }^{2}$, \\ Sergi Sànchez-López ${ }^{1}$, Marilia Curado ${ }^{2}$, Jordi Domingo-Pascual ${ }^{1}$ \\ ${ }^{1}$ Departament d'Arquitectura de Computadors, Universitat Politècnica de Catalunya (UPC) \\ Avgda. Víctor Balaguer, s/n - 08800 Vilanova i la Geltrú, Barcelona, Catalunya, Spain \\ \{yannuzzi, xmasip, sergio, jordid\}@ac.upc.es \\ ${ }^{2}$ Laboratory of Communications and Telematics, CISUC-DEI, University of Coimbra, \\ Pólo II, Pinhal de Marrocos, Postal Address 3030-290 Coimbra, Portugal \\ \{afonte, edmundo,marilia\}@dei.uc.pt \\ ${ }^{3}$ Polytechnic Institute of Castelo Branco, \\ Av. Pedro Álvares Cabral, $n^{\circ} 12$, Postal Address 6000-084, Castelo Branco, Portugal
}

\begin{abstract}
This paper proposes a novel and incremental approach to InterDomain QoS Routing. Our approach is to provide a completely distributed Overlay Architecture and a routing layer for dynamic QoS provisioning, and to use QoS extensions and Traffic Engineering capabilities of the underlying BGP layer for static QoS provisioning. Our focus is mainly on influencing how traffic is exchanged among non-directly connected multi-homed Autonomous Systems based on specific QoS parameters. We provide evidence supporting the feasibility of our approach by means of simulation.
\end{abstract}

Keywords: Inter-Domain QoS Routing, Overlay, BGP

\section{Introduction}

At present, nearly $80 \%$ of the more than 15000 Autonomous Systems (ASs) that compose the Internet are stub ASs [1], where the majority of this fraction is multi-homed. For these ASs the issue of Quality of Service Routing (QoSR) at the inter-domain level arises as a strong need [2]. Whereas some research groups rely on QoS and Traffic Engineering (TE) extensions to BGP [3-4], others tend to avoid new enhancements to the protocol and propose Overlay networks to address the subject [5-6]. While the former approach provides significant improvements for internets under low routing dynamics, the latter results more effective when routing changes occur more frequently. The main idea behind the overlay concept is to decouple part of the policy control portion of the routing process from BGP devices. In this sense, the two approaches differ in how policies are controlled and signaled. BGP enhancements tend to provide in-band signaling, while the overlay approach provides out-of-band signaling.

The Overlay Architecture is mostly appropriate when communicating domains are multi-homed, and thus may need some kind of mechanism to rapidly change their

\footnotetext{
${ }^{1}$ This work was partially funded by the MCyT (Spanish Ministry of Science and Technology) under contract FEDER-TIC2002-04531-C04-02, the CIRIT (Catalan Research Council) under contract 2001-SGR00226 and the European Commission through Network of Excellence E-NEXT under contract FP6-506869.
} 
traffic behavior depending on network conditions. In fact, multi-homing is the trend that most stub ASs exhibit in nowadays Internet, which mainly try to achieve load balance and fault tolerance on the connection to the network [5]. In addition, present inter-domain traffic characteristics reveal that even though an AS will exchange traffic with most of the Internet, only a small number of ASs is responsible for a large fraction of the existing traffic. Moreover, this traffic is mainly exchanged among ASs that are not directly connected; instead they are generally 2, 3 and 4 hops away [4].

Therefore, the combination of all these features made us focus on QoSR among strategically selected non-peering multi-homed ASs. The approach to inter-domain QoSR we propose in this paper is to supply a completely distributed Overlay Architecture and a routing layer for dynamic QoS provisioning, while we use QoS extensions and TE capabilities of the underlying BGP layer for static QoS provisioning. Within the overlay inter-domain routing structure reside special Overlay Entities (OEs), whose main functionalities are the exchange of Service Level Agreements (SLAs), end-to-end monitoring, and examination of compliance with the SLAs. These functionalities allow the OEs to influence the behavior of the underlying BGP routing layer, to take rapid and accurate decisions to bypass network problems such as link failures, or service degradation for a given Class of Service $(\mathrm{CoS})$. The reactive nature of this overlay structure acts as a complementary layer conceived to enhance the performance of the underlying BGP layer containing both QoS aware BGP (QBGP) routers and non-QoS aware routers.

The remaining of this paper is organized as follows. Section II presents an overview of our overlay approach. In Section III the main functionalities required from the underlying BGP and overlay layers are analyzed, while Section IV presents our simulation scenario and results. Finally, Section V concludes the paper.

\section{Overview of the proposed Overlay approach}

As stated in the Introduction, we propose in this paper a combined QBGP and Overlay Architecture for inter-domain QoSR. The main ideas behind the Overlay Architecture are:

- The OEs should respond nearly two orders of magnitude faster than the BGP layer in the case of a network failure.

- The OEs should react and try to reroute traffic when non-compliant conditions concerning QoS parameters previously negotiated for a given $\mathrm{CoS}$ are detected.

- The underlying BGP structure does not need modifications, and remains unaware of the QoSR architecture running on top of it.

The next figure depicts a possible scenario were our proposal could be applied. In this model, two peering OEs belonging to different ASs spanning across several AS hops are able to exchange a SLA and agree upon a set of QoS parameters concerning the traffic among them. The intermediate ASs do not need to participate in the Overlay Architecture, and therefore no OEs are needed within these transit ASs. From our perspective, the real challenge is to develop a completely distributed overlay system, where each OE behaves in a reflective manner. In this sense our overlay approach is like facing a mirror. Instead of proposing a complex scheme to dynamically and accurately manage how traffic enters a target AS, we focus on how traffic should exit from 


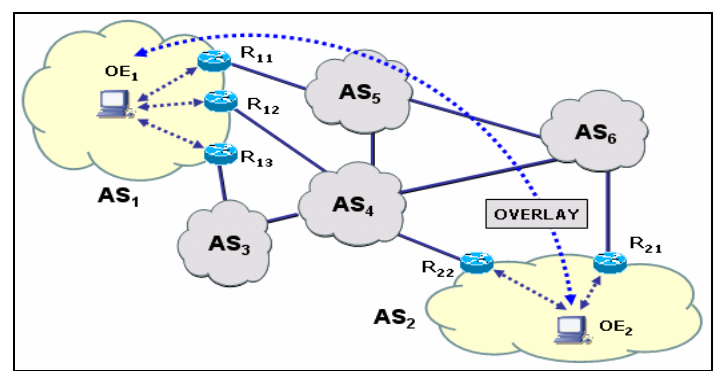

Fig. 1. Inter-Domain QoSR scenario where OEs are used for dynamic QoS provisioning among remote multi-homed ASs

the source AS. Hence, what we seek is that the OE within the source AS behaves like the image in a mirror of the OE in the target AS. This mirroring scheme allows the $\mathrm{OE}$ in the source AS to dynamically manage its outgoing traffic to the target AS, depending on the compliance with the previously established SLA for a given set of CoSs. Then, within each AS, the OE should measure end-to-end QoS parameters along every link connecting the multi-homed AS to the Internet, and check for violations to the SLAs. Henceforth, we assume that the topology has at least two different end-to-end paths between any pair of remote ASs participating in our QoSR model. When a violation is detected, the $\mathrm{OE}$ in the source AS is capable of reconfiguring onthe-fly its traffic pattern to the remote AS for the affected CoS. Here, the time scale needed to detect and react to a certain problem is very small when compared with the BGP time scale [7].

The end-to-end measurements are based on active AS path probing among peering OEs. Hence, each OE within an AS spawns probes targeting the remote AS through every available link connecting the source AS to the Internet. We sustain, and we will show by simulation that the AS-AS probing practice is not demanding neither in terms of traffic nor in terms of processing, as long as the number of overlay peering ASs and the number of CoSs remains limited. In fact, the traffic generated between two OEs is negligible. It is worth noting that a non-complying condition may only occur in a single direction of the traffic, which means that the bottleneck is merely on the upstream or the downstream path. For example, in Fig. 1 the OEs in $\mathrm{AS}_{1}$ and $\mathrm{AS}_{2}$ measure the same parameters, such as One-Way Delay (OWD) [8] or One-Way Loss (OWL) [9], and react in the same manner due to their mirrored behavior. Therefore, either of them is able to independently decide if it should shift its outbound traffic or not. An advantage of this approach is that BGP updates could be completely avoided if, for example, the LOCAL PREFERENCE (LOCAL_PREF) is used when reallocating this traffic.

Agarwal et al. proposed an interesting overlay mechanism to reduce the fail-over time and to achieve load balancing of traffic entering an AS [5]. However, this proposal does not reuse any QoS or TE capabilities from the BGP layer. Moreover, it introduces a centralized and complex server which allows an AS to infer, by means of heuristics, the topology and customer/peer relationships among the multiple ASs that conform all tentative paths known to any given peering AS in the overlay structure. The complexity introduced is mainly due to the fact that accurately controlling how traffic enters an AS is a very intricate task, particularly when this must be done dynamically. As an alternative, our proposal deals with the allocation of traffic from the 
source AS, since we strongly believe that simpler approaches such as this one will turn out more attractive to become deployed.

\section{Main functionalities of the routing layers}

In this Section we describe in detail the overlay routing functionalities as well as the underlay BGP routing functionalities.

\subsection{Top Layer: Overlay Routing Functionalities}

This layer is composed by a set of OEs:

\subsubsection{Basic set of components:}

- At least one OE exists per QoS domain.

- An OE has full access to the border BGP routers within an AS.

- An OE has algorithms for both detecting non-conforming conditions for a given $\mathrm{CoS}$, and deciding when and how to reallocate its traffic.

\subsubsection{Main components:}

An Overlay Protocol: A protocol between remote OEs is needed. This protocol allows OEs to exchange SLAs with each other, and to exchange substantial information for the Overlay Architecture.

Metric Selection: In order to validate our approach, we choose a simple QoS parameter for the dynamical portion of our QoSR model. The parameter we have selected is a smoothed OWD (SOWD), which defines the following metric:

$$
\overline{O W D}(m, n)=\frac{1}{N} \sum_{k=n}^{k=n+N-1} O W D(m, k)
$$

in which $m$ and $n$ correspond to the $n_{t h}$ probe generated by a source OE and sent towards the $m_{t h}$ external link of the AS. This SOWD corresponds to the average OWD through a sliding window of size $N$. Instead of using instantaneous values of the OWD, we propose to use this low-pass filter, which smoothes the OWD avoiding rapid changes in our metric. A trade-off exists in terms of the size of the window. A large value of $N$ implies a slow reaction when network conditions change and maybe the reallocation of traffic is needed. On the other hand, small values of $N$ could translate into frequent traffic reallocations since it is likely to occur that non-compliant conditions are more frequently met. In this scenario the SLA exchanged by the OEs is simply the maximum SOWD $D_{j}$ tolerated for each different $\operatorname{CoS} C_{j}$.

We assume that an $\mathrm{OE}$ uses one logical address for each different $\mathrm{CoS}$, and also that specific local policies are applied to Internal BGP (IBGP). Thus, a single OE could be configured to probe a remote $\mathrm{OE}$ for any given $\mathrm{CoS}$, through all available egress links of the AS in a round-robin fashion avoiding the hot potato routing problem. Then, the OEs compute a per-CoS cost to reach the remote AS over every external link $m$ based on the previous metric. Furthermore, packets probing a specific CoS belong to that CoS. For instance, in a QBGP framework based on Differentiated Services (DiffServ), when probing a particular CoS which is mapped to an Assured Forwarding (AF) class in each intermediate AS, the probes are tagged under the same AF class [10]. We assume that the OEs are properly synchronized (e.g., by means of GPS) and the details concerning synchronization are out of the scope of this work. 
Piggy-Backing mechanism: An important issue is that an active probing technique developed to measure the OWD requires feedback from the remote OE. However, the mirroring scheme implies that the remote OE is already probing the local OE and expects feedback from this latter as well. Thus, the easiest way to avoid unnecessary messages traversing the network is to endow the protocol between the OEs with a piggy-backing technique. Then, feedback for the OWD is carried on the probes itself.

Stability: Another central issue is that the traffic reallocation process should never generate network instability. In order to prevent this from happening, but keeping in mind that we follow a completely distributed architecture design where the OEs should rely on themselves to cope with these problems, we impose the following restriction:

"Traffic targeting a certain $\operatorname{CoS} C_{j}$ should never be reallocated over a link $s$, if and only if the primary link to reach $C_{j}$ was $s$ in $\left[t-T_{h}, t\right]$ or $C_{j}$ has exceeded its maximum number of possible reallocations $\Rightarrow R_{j}(t) \geq R_{j}^{\text {MAX }}$,

In this way the parameter $T_{h}$ avoids short-term bounces, while the parameter $R_{j}^{M A X}$ avoids the long-term ones. Then, each time a traffic reallocation process takes place for a given $\operatorname{CoS} C_{j}$ the variable $R_{j}(t)$ is incremented. Our approach is to provide a sort of soft penalization similar to BGP damping [11], where the penalty is incremented by a fixed value $P$ with each new allocation, but it decays exponentially with time when no reallocations occur according to:

$$
R_{j}(t)=R_{j}(T) e^{-\left(\frac{t-T}{\tau}\right)}
$$

where $T_{h}, R_{j}^{M A X}, P$ and $\tau$ are configurable parameters, whose values depend on the degrees of freedom in the number of short and long-term reallocations we allow for a given $\operatorname{CoS} C_{j}$. An additional challenge in terms of stability arises when a path becomes heavily loaded, since several CoSs within the path could experience noncompliant conditions with their respective SLAs. In order to prevent simultaneous reallocations for all the affected CoSs, we endow the OEs with a contention mechanism which prioritizes the relevance of the different CoSs. Then, more relevant CoSs are reallocated faster than less priority classes. The contention algorithm operates as follows:

$\left\{\right.$ Let $C_{j}$ be one of the $q$ affected $\operatorname{CoS}$ within link $m$, where $j=1, . ., q$

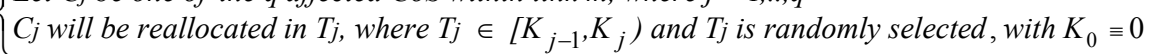

$\Rightarrow$ Then, the highest priority classes $C_{1}$ within link $m$ will be reallocated in a random time

$T_{1} \in\left[0, K_{1}\right)$, classes $C_{2}$ will be reallocated in a random time $T_{2} \in\left[K_{1}, K_{2}\right)$, and so on.

Clearly, our contention mechanism allows an OE to iteratively reallocate traffic from a loaded path, and to dynamically check if the remaining classes continue under non-compliant conditions. It is likely that as soon as we begin to extract traffic from the path, the remaining classes will start to experience better end-to-end performance. However, a different situation is generated when a link failure occurs. In this case, an OE should react as fast as possible to reallocate all traffic from the affected path. Then, a trade-off exists in terms of both the contention mechanism and the ability to rapidly redistribute all traffic from any given link. Instead of tuning the contention algorithm to efficiently cope with both problems at the same time, we rely on the probing technique since a link failure will cause the complete loss of probes for all the 
CoSs within the link. Our proposal is based on incrementing the frequency of the probes per-CoS as soon as losses are detected. We maintain that this rising in the frequency does not exacerbate the load on the network, firstly because the fraction of traffic generated by the $\mathrm{OE}$ that detects the problem is negligible in terms of the overall traffic exchanged between both ASs. Secondly, this is done for a short period of time and only with the aim of speeding up the re-routing process. Once a CoS is reallocated, the frequency of the probes decreases back to its normal value.

\subsection{Bottom Layer: Underlay BGP Routing Functionalities}

The set of routes to be tested by the OEs using the probing techniques described in the previous sub-section, are predetermined by the underlying BGP-based layer. In this layer two types of devices can operate; legacy BGP routers and QBGP routers. A QBGP router is able to distribute QoS information and take routing decisions per-CoS constrained to the previously established SLA between different peering domains. In our model, QBGP routers can be seen as the practical tool to establish the overall inter-domain QoSR infrastructure composed by several sub-routing layers, one for each $\mathrm{CoS}$, which in addition could be dynamically influenced by the overlay layer. Interesting approaches and further information on the subject of QBGP could be found in $[3,12]$.

\subsection{Combined QoSR Algorithm}

The next scheme (Fig.2) depicts our combined QoSR algorithm. Let $m$ be the external link currently allocating traffic of class $C_{j}$. It is important to remark that the approach we follow is that even though an alternative path could have a better cost in terms of SOWD, we avoid reallocating traffic of class $C_{j}$ from link $m$ until a violation to the SLA is detected. Then, two distinct threads of events occur upon the reception of a probe for class $C_{j}$. Initially, the probe $(k, l)$ is separated from the piggy-backed feedback $O W D(m, n)$. In order to accurately reply back to the sender, the first to be processed is the $O W D(k, l)$ which is shown as (I) in Fig. 2. On the other hand, the piggy-backed $\operatorname{OWD}(m, n)$ is processed, which is depicted as (II) in the figure.

Once the SOWD is computed, the algorithm checks for violations to the maximum SOWD tolerable, that is $D_{j}$. If no violations have occurred the algorithm simply waits for the next incoming probe. However, if a violation is detected in link $m$ the algorithm checks if the maximum number of allowed reallocations $R_{j}^{M A X}$ is exceeded. In case this is true, the local OE is able to compose a feedback message and warn the remote $\mathrm{OE}$ about this situation. The main idea is that the feedback process provides information to the remote $\mathrm{OE}$, and thus it can try to handle the problem by tuning its static QoS provisioning using either QBGP or TE-BGP.

If $R_{j}^{M A X}$ is not exceeded, then the OE needs to check, within all the external available links $p$, excepting $m$, if there exists at least one link $i$ whose $\operatorname{cost} M_{i}$ satisfies the constraint for the class $C_{j}$. Moreover, it also needs to check if the link has enough room to handle the class reallocation. Subsequently, and in order to avoid any short term bounce, the OE excludes from the set of capable links those who had allocated traffic of $C_{j}$ in $\left[t-T_{h}, t\right]$. Once this is done, we rely on QBGP to tiebreak in case two or more links show the same cost in terms of the SOWD. At this step a single link is left as the target for the reallocation of the class. Then, the contention algorithm is executed and $T_{j}$ seconds later the OE checks if the class still remains in a violating condition. If this is the case, the $\mathrm{OE}$ increments $R_{j}(t)$ by $P$ and reroutes the traffic of $C_{j}$. 


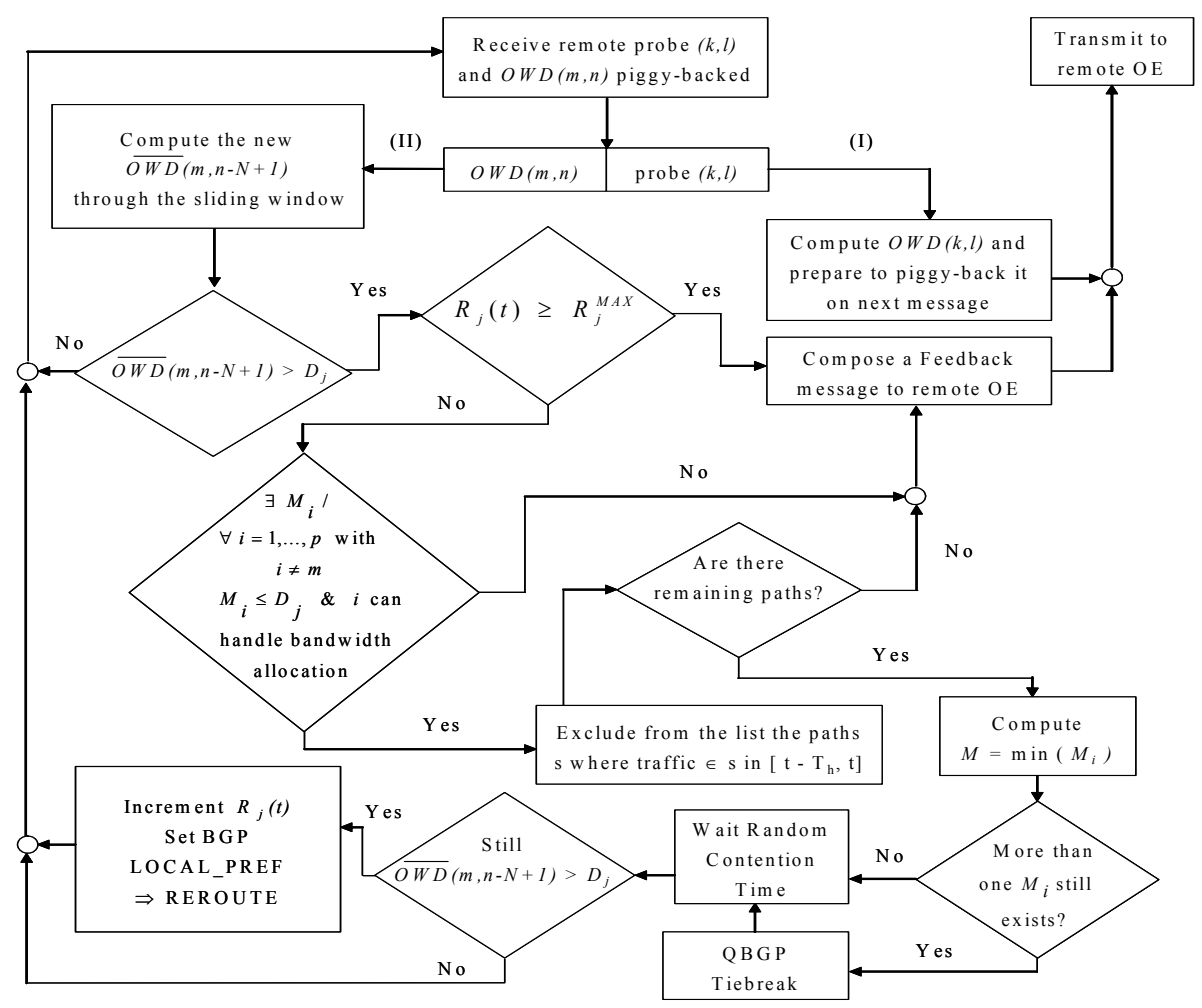

Fig. 2. Combined QoSR Algorithm

\section{Simulation Results}

The Overlay Architecture proposed in this paper is being evaluated and validated by simulation. In this section some preliminary results are presented to allow a first evaluation of the overall architecture and its capability to support QoS traffic classes in a dynamic way. We are using the J-Sim simulator [13] with the BGP Infonet suite [14] which is compliant with BGP specification RFC 1771 [15]. A set of Java components with the functionalities of the overlay layer was developed. In order to allow the Overlay Entities to have full access to the Adj-RIBs-In and the Loc-RIB of a BGP speaker [15], and to have control over the BGP decision process, it was necessary to add some extensions to the Infonet suite. Furthermore, we have also included the following QoS BGP extensions:

- An optional transitive attribute to distribute the CoS identification (ID), and a set of modifications to BGP tables to allow the storage of this additional information, following a similar approach to the one described in [3].

- A set of mechanisms to: i) allow BGP speakers to load the supported CoSs; ii) allow each local IP prefix to be announced within a given CoS; iii) allow BGP speakers to set the permissibility based on local QoS policies and supported capabilities.

For our simulations, we used the topology presented in Fig. 7. The topology is 
based in the GÈANT European Academic Backbone with some simplifications to reduce the complexity of the simulation model. In this topology we considered as remote multi-homed AS domains $\mathrm{AS}_{1}$ and $\mathrm{AS}_{2}$. All links were assumed to be bidirectional with the same capacity $\mathrm{C}(\mathrm{C}=2 \mathrm{Mbps})$ and propagation delay $\mathrm{P}_{\mathrm{d}}\left(\mathrm{P}_{\mathrm{d}}=10 \mathrm{~ms}\right)$, with the exception of $\mathrm{AS}_{2}$ links where, in order to have some bottleneck, the capacity chosen was $\mathrm{C} / 2$. For complexity concerns, we modeled each AS as a single QBGP router with core DiffServ capabilities configured to support four different IP packet treatments (EF, AF11, AF21 and Best-effort) allowing four different CoSs, namely $\operatorname{CoS} 1, \operatorname{CoS} 2, \operatorname{CoS} 3$ and CoS4. Thus, on the domain where traffic was injected we used edge DiffServ capabilities to mark packets with a specific DSCP (DiffServ Code Point) depending on its corresponding CoS. These marks were applied to regular IP packets and to the probes generated by the OE. The test conditions are summarized in Table 1. The results obtained are presented in Fig. 3 to Fig. 6. The maximum SOWD tolerated per-CoS $\left(D_{j}\right)$ was heuristically chosen to allow the OEs to take advantage of alternative paths. The SWOD computed when probes were lost was also heuristically chosen. The criterion selected was that 3 consecutive losses imply nearly a rise of $25 \%$ in the SWOD. For the tests presented we set $R_{j}^{M A X}=\infty \forall j$. Moreover, no probes were generated for Best-effort traffic ( $\mathrm{NA}=$ Not Available), and a sliding window of 3 seconds was used in all tests, which is shown as Mov.Average in Table 1.

Table 1. Test conditions

\begin{tabular}{lccccccc}
\hline CoS & $\begin{array}{c}\text { CBR } \\
(\mathbf{M b p s})\end{array}$ & $\begin{array}{c}\text { Pkt. Size } \\
(\mathbf{K B})\end{array}$ & PHB & $\begin{array}{c}\text { Max. SOWD } \\
(\mathbf{m s})\end{array}$ & $\begin{array}{c}\text { Probing } \\
\text { Freq. }\end{array}$ & $\begin{array}{c}\text { Hold (Contention) } \\
\text { \& }_{\mathbf{h}}(\mathbf{s})\end{array}$ & $\begin{array}{c}\text { Mov. } \\
\text { Average }\end{array}$ \\
\hline CoS1 & 0,4 & 1 & EF & 85 & $1 \mathrm{~s}, 1 \mathrm{~KB}$ & $3 \& 8$ & $3 \mathrm{~s}$ \\
\hline CoS2 & 0,8 & 1 & AF11 & 100 & $1 \mathrm{~s}, 1 \mathrm{~KB}$ & $6 \& 12$ & $3 \mathrm{~s}$ \\
\hline CoS3 & 1,0 & 1 & AF21 & 120 & $1 \mathrm{~s}, 1 \mathrm{~KB}$ & $9 \& 20$ & $3 \mathrm{~s}$ \\
\hline CoS4 & 1,6 & N.A & BE & N.A & N.A & N.A & N.A \\
\hline
\end{tabular}

The first objective of the simulation was the validation of the initial assumption that our approach, based on a complementary routing layer, enhances the reaction of the overall routing infrastructure. Then, as a performance indicator, we chose to compare the response time to a link failure. Fig. 3 depicts a set of plots for traffic of CoS1 showing the throughput measured at the destination, the SOWD experienced by probes for all available paths, and the path shifts determined by changes in the nexthop for the source AS, namely $\mathrm{AS}_{1}$. From these plots, we can observe that a pure QBGP framework (without OEs running on $\mathrm{AS}_{1}$ and $\mathrm{AS}_{2}$ ) needs about 80 seconds to
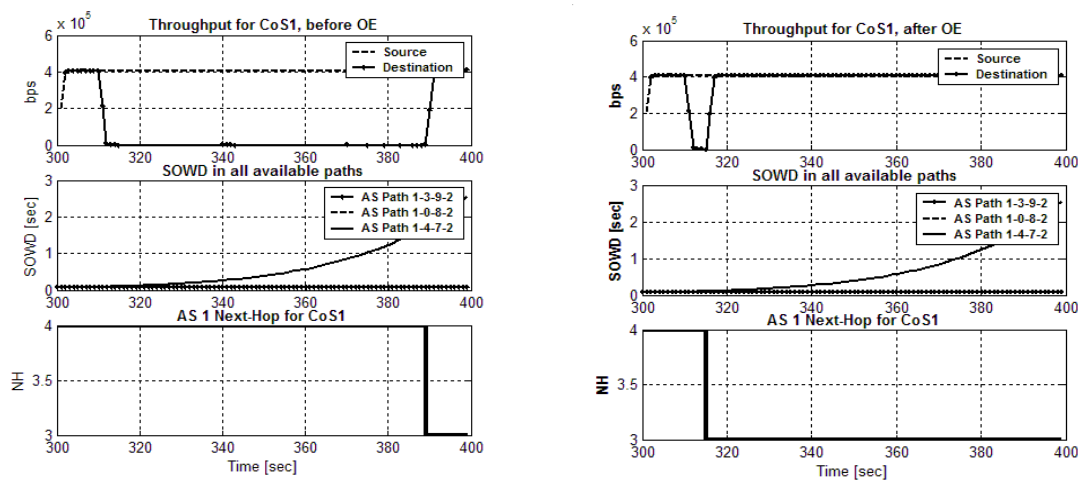

Fig. 3. Link failure reaction with and without $\mathrm{OE}$ 
overcome a link failure, but only 5 seconds are needed when OEs are running. This result validates our initial assumption. It is worth mentioning that this last value includes not only the implicit link failure detection condition based on a violation to the maximum SOWD tolerated, but also includes a random contention interval of 3 seconds before re-routing.

Secondly, from figures 4 and 5, we can observe that without OEs there are clear violations to the SLAs established between the end-to-end domains. However with OEs, it becomes clear that the architecture is able to react to SLA violations, and find the best paths to reallocate traffic for the affected traffic classes. Consequently, after a transitory interval of approximately 13 seconds, needed to accommodate the traffic for each $\mathrm{CoS}$, it is visible that a steady state is reached and the SLAs are satisfied for all affected classes. Furthermore, and in order to evaluate overall link utilization, we measured the throughput over all available links at the destination $\mathrm{AS}\left(\mathrm{AS}_{2}\right)$. Fig. 6 shows that with OEs, in addition to the compliance with the SLAs a better distribution
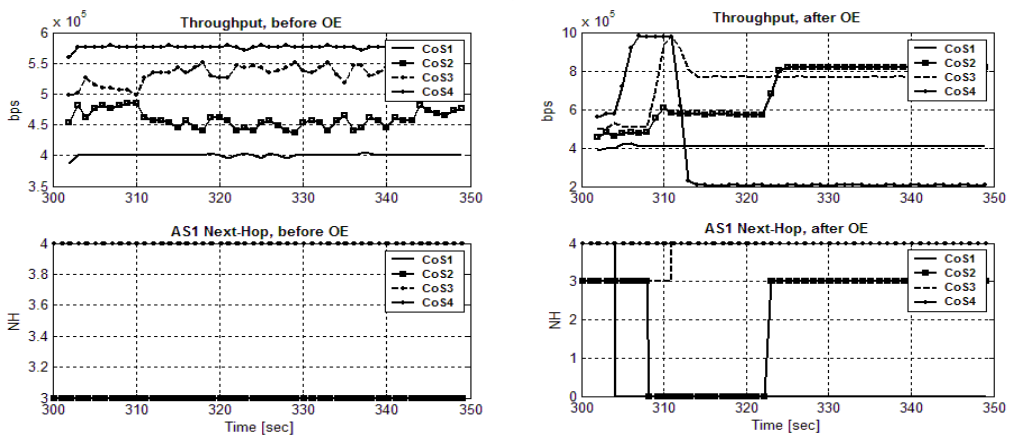

Fig. 4. Throughput for traffic of $\operatorname{CoS} 1-\operatorname{CoS} 4$, with and without $\mathrm{OE}$
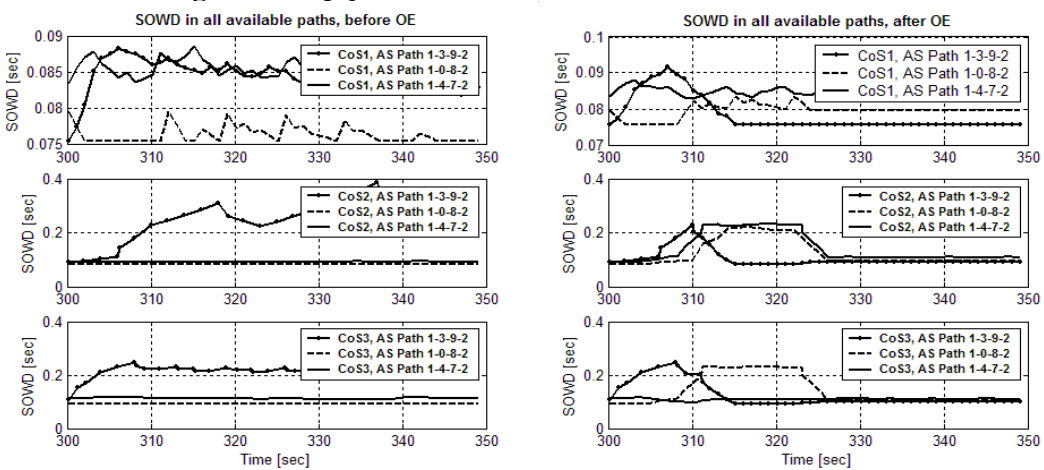

Fig. 5. OWD in all available paths for $\operatorname{CoS} 1-\mathrm{CoS} 4$, with and without OE

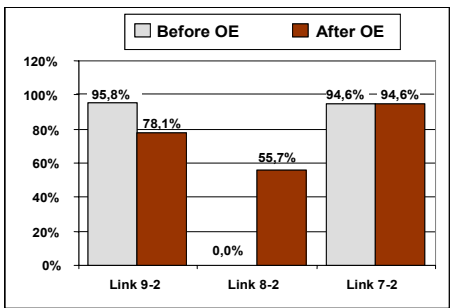

Fig. 6. Remote AS link utilization

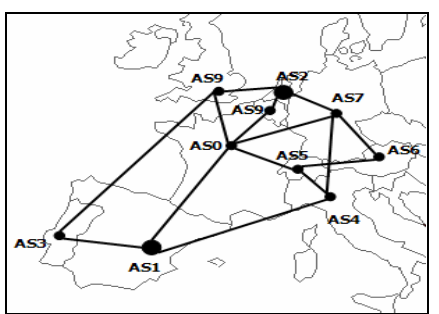

Fig. 7. Topology based on the GÉANT Network [16] 
of inter-domain traffic is obtained, and thus, resources are more efficiently used. The extra cost in these cases was merely an increment of $8 \mathrm{Kbps}$, per-CoS, on each link in the remote AS-AS traffic, when oversized probes of $1 \mathrm{~KB}$ were spawned.

\section{Conclusions}

This paper depicts the framework for a combined inter-domain QoSR paradigm based on a completely distributed Overlay Architecture coupled with a QBGP or TEBGP routing layer. As a first step in our research, and in order to validate our approach we have focused on the coupling of the overlay with a DiffServ QBGP underlying layer. The results obtained show that our distributed Overlay Architecture substantially enhances end-to-end QoS when compared with a pure QBGP model. We believe that whereas significant extensions and enhancements to BGP are certainly going to be seen, the overlay structure arises as a strong candidate to provide flexible and value-added out-of-band inter-domain QoSR. In particular, this becomes perfectly suitable when inter-domain traffic patterns need to dynamically adapt and rapidly react to medium or high network changing conditions, where the former solutions seem impracticable at the present time.

\section{References}

1. Olivier Bonaventure, Bruno Quotin, Steve Uhlig, "Beyond Interdomain Reachability", Workshop on Internet Routing Evolution and Design (WIRED), October 2003.

2. E. Crawley, R. Nair, B. Rajagopalan, H. Sandick, "A Framework for QoS-based Routing in the Internet", Internet Engineering Task Force, Request for Comments 2386, August 1998.

3. Cristallo, G., C. Jacquenet, “An Approach to Inter-domain Traffic Engineering”, Proceedings of XVIII World Telecommunications Congress (WTC2002), France, September 2002.

4. Olivier Bonaventure, Steve Uhlig, Bruno Quotin, et al, "Interdomain Traffic Engineering with BGP”, IEEE Communications Magazine, May 2003.

5. S. Agarwal, C. Chuah, R. Katz "OPCA: Robust Interdomain Policy Routing and Traffic Control", IEEE Openarch, April 2003.

6. Zhi Li, Prasant Mohapatra, "QRON: QoS-aware Routing in Overlay Networks", IEEE Journal on Selected Areas in Communications, June, 2003

7. C. Labovitz, A. Ahuja, A. Bose, and F. Jahanian, "Delayed Internet routing convergence," in Proc. ACM SIGCOMM, 2000.

8. G. Almes, S. Kalidindi, M. Zekauskas, "A One-way Delay Metric for IPPM”, Internet Engineering Task Force, Request for Comments 2679, September 1999.

9. G. Almes, S. Kalidindi, M. Zekauskas, "A One-way Packet Loss Metric for IPPM", Internet Engineering Task Force, Request for Comments 2680, September 1999.

10. J. Heinanen, F. Baker, W. Weiss, J. Wroclawski, “Assured Forwarding PHB Group”, Internet Engineering Task Force, Request for Comments 2597, June 1999.

11. C. Villamizar, R. Chandra, R. Govindan, "BGP Route Flap Damping", Internet Engineering Task Force, Request for Comments 2439, November1998.

12. IST MESCAL project, "Specification of Business Models and a Functional Architecture for Inter-domain QoS Delivery", Deliverable D1.1, June 2003.

13. J-Sim Homepage, http://www.j-sim.org.

14. Infonet Suite Homepage, http://www.info.ucl.ac.be/ bqu/jsim/

15. Y. Rekhter, T. Li , "A Border Gateway Protocol 4 (BGP-4)", Internet Engineering Task Force, Request for Comments 1771, March 1995.

16. GÉANT Website, http://www.dante.net/server/show/nav.007 